\title{
Intervención de Terapia Ocupacional en Síndrome de Inmovilización instaurado
}

BARRERA GUÍ ÑEZ, LOI DA ( 1). SI LVA CONCHA, LUZMARI NA ( 2)

(1) Terapeuta Ocupacional, Universidad de Chile; Licenciada en Ciencias de la Ocupación. Diplomada en Geriatría y Gerontología INTA. Profesor Asistente Universidad de Playa Ancha, Carrera de Terapia Ocupacional, Facultad de Educación Física, Departamento de Salud y Rehabilitación.(2)Terapeuta Ocupacional, Universidad de Chile; Magíster en Gerontología Social @ , Universidad de Valparaíso. Diplomada en Geriatría y Gerontología INTA. Profesor Instructor Universidad de Playa Ancha, Carrera de Terapia Ocupacional, Facultad de Educación Física, Departamento de Salud y Rehabilitación

Contacto > > Fono: 32- 500183 Email: loidabarrera@yahoo.es. Fono: 32- 500183 Email: Isilva@upa.cl

Referencia > > Loida Barrera Guíñez. LuzMarina Silva Concha. "Intervención de Terapia Ocupacional en Sindrome de Inmovilización instaurado". Revista Chilena de Terapia Ocupacional. №5, Noviembre 2005

\section{- Abstract}

The immobilization syndrome causes to manifold complications in the greater people being the motor complications, those that cause greater difficulty in their handling, reason why the environmental design of adapted equipment and modifications on the part of the occupational therapist, facilitate the handling and comfort of the patient, as well as, facilitates the task to the caretaker to position and the rest of the professional equipment. The present I articulate analyzes the intervention of the occupational therapist and it within the framework bases it theoretical of rehabilitador reference.

\section{Resumen}

El síndrome de inmovilización causa múltiples complicaciones en las personas mayores siendo las complicaciones motoras las que ocasionan mayor dificultad en su manejo, por lo que el diseño de equipo adaptado y las modificaciones ambientales por parte del Terapeuta ocupacional, facilitan el manejo y confort del paciente, así como la tarea al cuidador a cargo y al resto del equipo profesional. El presente artículo analiza la intervención del Terapeuta Ocupacional y lo fundamenta en el marco teórico de referencia rehabilitador.

\section{- Introducción "}

El deterioro de la movilidad es el problema más frecuente experimentado por los adultos mayores (AM) ingresados en instituciones. ${ }^{(1)}$

En el AM se prolongan los periodos de reposo en cama, tanto como respuesta tradicional al proceso de enfermedad como por la creencia de que se trata de un medio idóneo para mitigar el dolor, ciertas incapacidades e incluso el aburrimiento y como expresión de celo, "el lugar más seguro", en clara contradicción con los planteamientos actuales conocidos los efectos nocivos del encamamiento. 
Los efectos del deterioro prolongado de la movilidad en los AM pueden ser irreversibles, provocando una pérdida permanente de la función.

El síndrome de inmovilidad es un problema geriátrico caracterizado por una reducción marcada de la tolerancia al ejercicio (respuesta taquicardizante, hipertensión arterial, disnea...), progresiva debilidad muscular y, en casos extremos, pérdida de los automatismos y reflejos posturales que imposibilitan la deambulación (2). Dentro de éste puede distinguir dos niveles:

- Una inmovilidad relativa, en la que el anciano lleva una vida sedentaria pero es capaz de movilizarse con menor o mayor independencia.

- Una inmovilidad absoluta que implica el encamamiento crónico, estando muy limitada la variabilidad postural.

\section{- Complicaciones asociadas a la inmovilidad que involucran al sistema locomotor. "}

La literatura en general hace mención al síndrome de inmovilización centrado en la prevención y tratamiento para el retorno a la movilidad, pero lamentablemente existe un porcentaje de adultos mayores que se encuentran en estado de postración irreversible, con un patrón postural alterado con los consecuentes problemas de manejo sanitario para sus cuidadores ${ }^{(1)}$.

Una vez instaurado, trae como consecuencia:

- Limitación de los rangos de movimiento (pasivo)

- Disminución de la amplitud de movimiento (activo)

- Contractura muscular

- Posturas viciosas

- Dolor a la movilización

- Edema

- Dificultad para la higiene y procedimientos de enfermería

- Lesiones a la piel (micosis, maceración, ulceración, etc.)

Se observa generalmente en extremidades superiores un compromiso bilateral, siendo en ocasiones más predominante en una extremidad que la otra. La postura es la siguiente:

- Aducción y rotación interna de hombros, flexión de codos y manos empuñada con aducción de pulgar, el que puede estar o no incluido. La posición de las muñecas y de los antebrazos es variable.

En tanto, en extremidades inferiores se observa dos patrones con mayor frecuencia:

- Patrón flexor: flexión y aducción de caderas, flexión de rodillas, pies en equino.

- Patrón extensor: Caderas en semiflexión, rodillas en extensión, pies en equino e inversión plantar, pudiendo en ocasiones agregarse aducción de caderas, que lleva las extremidades a un entrecruzamiento.

Además, se suma el hecho de su compromiso cognitivo que impide la comprensión y colaboración en su tratamiento. Por lo tanto, las medidas a adoptar sólo son instrumentales o paliativas en base a la modificación del entorno, equipamiento adaptado y de asistencia. 


\section{- Marco teórico de referencia -}

La intervención del Terapeuta Ocupacional en personas que presentan un Síndrome de inmovilización instaurado se justifica bajo el Marco de Referencia de Rehabilitación o Compensatorio, el cual propone una intervención que "no pretende cambiar las carencias biológicas, fisiológicas o psicológicas"(곤, (4) sino facilitar el desempeño en diversas actividades de la vida diaria mediante la adaptación de las actividades o a través de diferentes métodos compensatorios como son las modificaciones ambientales, el equipamiento adaptado y de asistencia $^{(3)}$.

Es así como "el equipamiento adaptado y de asistencia pueden ayudar a las personas a facilitar su cuidado personal, mejorar el mantenimiento de la salud y prevenir la deformidad"(3).

\section{- Intervención de Terapia Ocupacional "}

\section{EVALUACIÓN}

- Anamnesis:

Es fundamental realizar una historia clínica detallada, debiendo recurrir frecuentemente a familiares y cuidadores para completar datos. Deben incluirse los apartados siguientes:

a) Forma de aparición y grado de inmovilidad. Debe indagarse desde cuando está inmovilizado y la repercusión sobre las actividades básicas e instrumentales de la vida diaria y sobre las recreativas.

b) Historia farmacológica. Es necesario que todo el equipo que atiende al paciente conozca los motivos de prescripción, duración, dosis. Esto es especialmente importante para determinados fármacos que pueden alterar la movilidad, como los antihipertensivos, los psicofármacos (neurolépticos, benzodiacepinas, antidepresivos) y los antihistamínicos. se ha de realizar una cuidadosa evaluación del potencial de riesgo/ beneficio para su mantenimiento o no. Es una buena norma a seguir que el fármaco que no es indispensable debe ser retirado.

- Detectar factores de riesgo para la inmovilidad.

Es necesario evaluar la presencia de patología crónica, estado mental, visión y audición, estado nutricional, historia previa de caídas, antecedentes de fracturas, enfermedad aguda reciente, cambio de domicilio o antecedentes de ingresos hospitalarios recientes.

- Analizar los factores psicosociales.

Hay que valorar la edad, estado civil, nivel educativo, nivel socioeconómico, entorno social y familiar, ya que estos factores pueden condicionar o agravar un problema de movilidad. El conocimiento de los recursos sociales (relaciones sociofamiliares, grado de apoyo social formal e informal) se puede conseguir mediante escalas de valoración como el OARS. 
- Observación:

Postura habitual del paciente en cama, silla de rueda o sillón de descanso.

Deformidades o posturas viciosas

Prominencias óseas, zonas de descarga de peso, signos de enrojecimiento.

- Edema.

Conexión con el entorno.

- Evaluación de Funciones cognitivas.

Pueden ser abordadas a través de pautas estandarizadas de evaluación del estado mental ( mini mental, escala de Pfeiffer, etc) o a través de una observación no estructurada de conexión con el entorno y orientación témporo- espacial.

- Movilidad

Movilización pasiva de las extremidades

Movilidad activa, en caso que proceda

Deformidades reductibles y no reductibles

Tono muscular predominante de los principales grupos musculares.

- Entrevista con el cuidador

Rutina del paciente

Principales dificultades en las actividades de la vida diaria.

- Valoración del entorno físico

Dormitorio: ubicación dentro de la casa, tipo de cama, alzarropa, tipo de colchón, mesa de cama, velador, acceso desde y hacia otras habitaciones.

Baño: Tina o receptáculo ducha, ducha teléfono, barras de sujeción, antideslizante, silla de baño, espacio libre de circulaciónlluminación y ventilación: luz directa e indirecta, ventanas cercanas.

\section{2.- OBJETIVOS}

Los objetivos de intervención propuestos S

- Mantener alineamiento corporal

- Mantener rangos articulares pasivos y/o activos

- Facilitar higiene y procedimientos de enfermería

- Prevenir úlceras por presión y edema

\section{3.- MÉTODO DE INTERVENCIÓN}

Para lograr el primer objetivo antes expuesto es necesario considerar los siguientes elementos dentro las modificaciones ambientales ${ }^{(1)}$ : 
- CAMA : Es indispensable que cuente con pie y cabecera, ya que facilita la movilidad del usuario. Para las personas que deben permanecer largos períodos en cama debido a su movilidad restringida, se debe disponer de un catre clínico, con algún tipo de colchón antiescaras, ya sea este de gel, de presiones alternantes o espuma plástica (moltopren). En caso de no contar con un catre clínico, la cama común deberá ser adaptada elevando su altura con tacos de madera u otro material resistente, ya que esto facilitara las transferencias y los cambios posturales para quien ejerce la labor de cuidador.

- VELADOR: Debe ubicarse a un costado de la cama y estar nivelado con la altura de ésta para facilitar el acceso desde la misma. Su función es la de guardar algunos artículos personales (pañuelos, artículos de tocador, medicamentos, etc.), sirve además de apoyo para la lámpara y eventualmente alguna fotografía o artículo personal decorativo que sea de especial significado para el paciente. Por lo tanto debiera evitarse el exceso de otros elementos que entorpezcan su función.

- MESA AUXILIAR: Se utiliza como superficie de alimentación o trabajo. Debe ser móvil (con ruedas y liviana) para poder desplazarla en los momentos que el paciente no la esté utilizando. Su altura debe ser en lo posible regulable, para adaptarla según el paciente.

- ILUMINACIÓN: Se puede poner una luz permanente de baja intensidad que permita al anciano orientarse si se despierta durante la noche. Si la lámpara está situada sobre el velador, es conveniente fijarla para que no se caiga al encender o apagarla.

Para el logro de los tres últimos objetivos se propone el uso de los siguientes elementos terapéuticos y equipamiento de asistencia:

- FÉRULAS: Utilizados como elemento posicionador de extremidades inferiores y extremidades superiores, suelen ser confeccionadas en termoplástico por su condición de higiene y remoldeo.

Para extremidades superiores lo más frecuente es la utilización de palmetas de reposo y rollos cónicos en aquellas personas que por sus retracciones no logran la extensión de los dedos simultáneamente a la dorsiflexión de muñeca. En extremidades inferiores lo más frecuente es la valva antiequino que puede ser de apoyo posterior o anterior con base plantar, según el grado de espasticidad del paciente.

- COJINES: Los cojines posicionadores existentes en el comercio son en su mayoría estandarizados, se compran por catálogos y tallas y como su fin es comercial, se encuentran solo aquellos de uso más frecuente, como por ejemplo picarones, taloneras, cuñas, colchones y cojines antiescaras.

Pero la complejidad del cuidado crea la necesidad de diseñar y confeccionar algunos aditamentos más específicos para mantener el alineamiento y la amplitud articular mínima que permita movilizar al paciente en sus actividades de la vida diaria.

Estos aditamentos deben ser diseñados bajo las siguientes características:

- Anatómicos

- Bajo costo

- Fácilmente reproducibles

- Confeccionados en un material que no dañe la piel (textura suave, hipoalergénicos, que permitan la ventilación)Flexibles y adaptable

- Lavables.

a) Picarones: Confeccionados en espuma plástica de mediana o alta densidad, se adquieren

fácilmente en casas comerciales especializadas en enfermos. Su uso es prescrito para aliviar la descarga de peso de prominencias óseas como la región sacra, talones, codos, etc.

b) Cuñas : Confeccionados en espuma plástica de mediana o alta densidad, son bloques de esponja plástica cortados con una pendiente de $45^{\circ}$, de largo y ancho variable según el segmento a apoyar (extremidades 
superiores, una o ambas extremidades inferiores). Su uso principal es destinado a elevar segmentos para facilitar el drenaje de las extremidades edematosas, sin embargo la experiencia nos ha demostrado su utilidad como apoyo posterior para mantener en cama la posición decúbito lateral y facilitar la posición sedente como apoyo para la espalda.

Para aquellos pacientes que tienden a deslizarse hacia delante al estar en sedente, ya sea en silla de ruedas o silla común, una cuña cortada según el ancho del asiento y dispuesta sobre él con la cara más alta bajo el hueco poplíteo, posiciona las caderas en flexión de $100^{\circ}$ o más, evitando así el deslizamiento.

c) Bloques de esponja: Confeccionados en espuma plástica de mediana o alta densidad, suelen ser de forma cuadrilátera y tamaño variado de acuerdo a su propósito. Lo más común es que se utilicen para mantener el alineamiento de tronco en sedente y para separar zonas de contacto en extremidades inferiores (cóndilos internos, maleolos internos) al poner en decúbito lateral (fotos 1y 2).

Foto 1

Patrón postural, en decúbito lateral

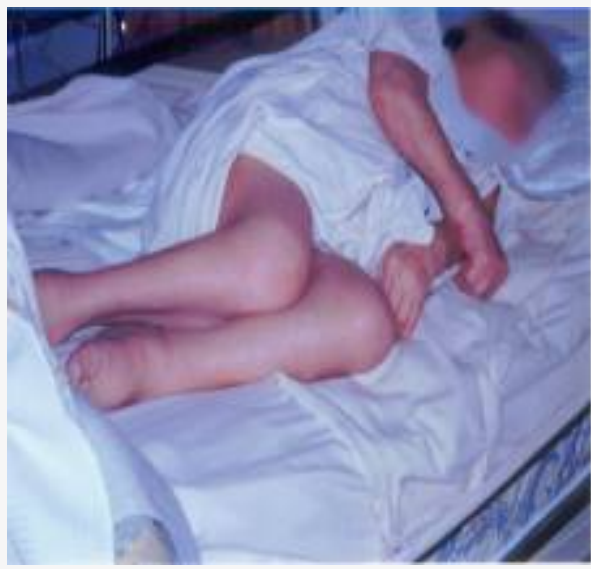

Foto 2

Cojín de bloque, aliviando zonas de presión

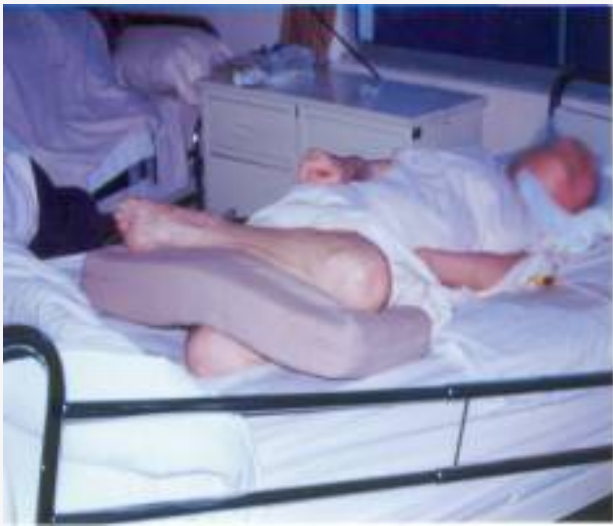

Dentro de esta categoría haremos mención a dos cojines diseñados para posicionar zonas de difícil acceso en personas que presentan patrón flexor en extremidades inferiores y superiores. Para efectos prácticos los hemos denominados "Cojín T" y "Cojín L".

\section{Cojin T}

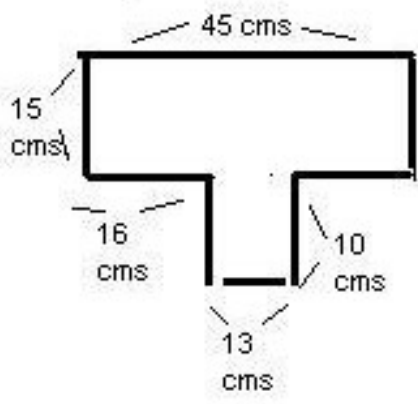

\section{Cojín L}

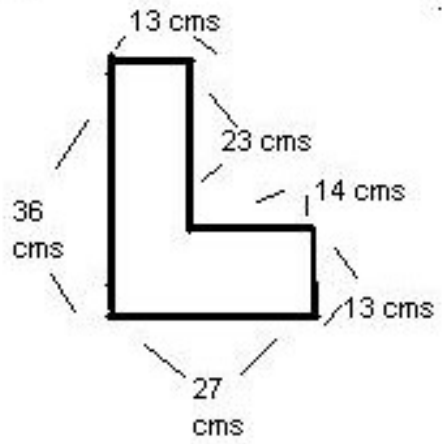




\section{Cojín T:}

Confeccionado en espuma plástica de mediana densidad, es un bloque que asemeja a la letra "T", en cuanto se trata de un rectángulo de $10 \mathrm{cms}$. de espesor, de $45 \times 15 \mathrm{cms}$. que en el centro lleva un brazo perpendicular de $13 \times 10 \mathrm{cms}$. Su función es contener la flexión de la pierna contra el muslo y a la vez evitar la formación de úlceras por presión en la cara interna de las rodillas, particularmente durante la posición decúbito lateral. Este debe ubicarse apoyando la zona mas larga en el pliegue poplíteo y la zona mas corta y perpendicular entre ambos cóndilos internos, separando así las rodillas (fotos 3 y 4).

Foto 3

Paciente en decúbito lateral, con evidentes zonas de presión

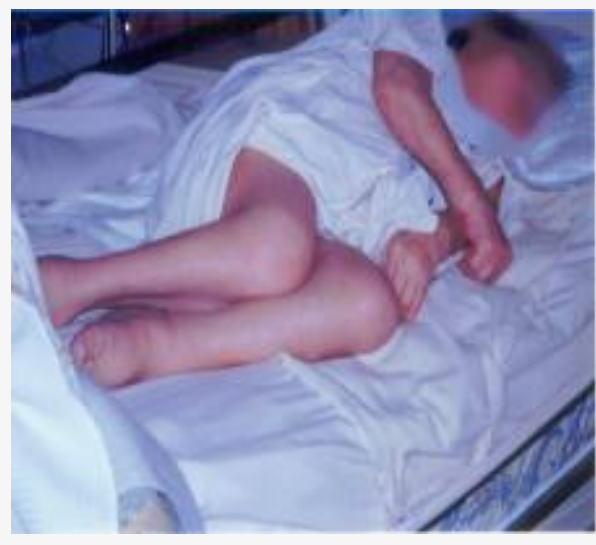

Foto 4

Cojin T. corrigiendo postura

\section{Cojín L:}

Confeccionado en espuma plástica de mediana densidad de $10 \mathrm{cms}$. de espesor, es un bloque cortado en ángulo recto, con dos brazos, siendo el de mayor longitud $36 \times 13 \mathrm{cms}$, y el otro de $27 \times 13 \mathrm{cms}$. Su función es abducir levemente el hombro, a la vez que proporciona apoyo para el brazo y el antebrazo, separándolo del tórax, facilitando la higiene de la axila y protegiendo las mamas de la compresión y micosis. Debe ubicarse uno de los brazos del cojín bajo el pliegue axilar, de modo que el ángulo quede a la altura del codo, posicionando el antebrazo sobre el otro extremo.

Sacos de Arena: Se utilizan para evitar la rotación interna o externa de extremidades inferiores y el entrecruzamiento de éstas. Estos deben ser sellados en una funda plástica y sobre ésta forrada con un género de algodón acolchado o tela de "chiporro", para evitar lesiones en la piel (foto 5). 


\section{Foto 5}

Sacos de arena manteniendo alineamiento de EEII

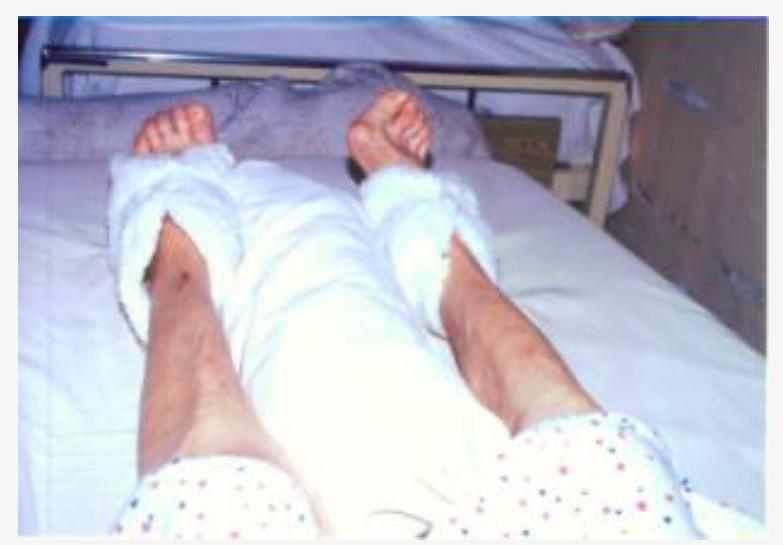

Cabe además mencionar que un aspecto fundamental en el éxito de intervención del Terapeuta Ocupacional en personas con síndrome de inmovilización instaurado lo constituye la educación de quienes ejercen la labor de cuidador directo, ya que serán ellos los encargados utilizar el equipamiento en cada cambio de posición del paciente. Esta labor educativa incluye dos aspectos:

- Entrenamiento directo de la correcta postura de los equipos de asistencia para que cumplan efectivamente con la finalidad para la cual fueron prescritos.

- Educación en la utilidad y cuidados del equipamiento para asegurarnos de la continuidad en el uso y prolongación de su vida útil.

\section{Conclusión}

Generar programas de animación socio-cultural es una responsabilidad para quienes trabajamos día a día por el bienestar ocupacional de los adultos mayores. En este sentido las acciones que fundamentan este tipo de programas merecen el análisis y el conocimiento de las posibilidades de participación y de los servicios ofrecidos por entidades públicas y privadas. El uso productivo del tiempo libre en cualquier etapa del ciclo vital es y seguirá siendo objeto de estudio de la Terapia Ocupacional, sin embargo en la población adulta mayor es una necesidad inminente conceptualizar en torno a su significado y a lo que este representa en la vida cotidiana de estas personas, reconociendo la importancia de reflexionar minuciosamente sobre los intereses, valores, contextos, materiales, historia ocupacional, hábitos, motivaciones, como factores que garantizan la realización exitosa del programa a desarrollar. 


\section{" Referencias "}

(1) ELIZASU, C. La animación con personas mayores. Editorial CCS. Madrid. 1999. Pág. 12 13 y $54-55$

(2) ELIZASU, C. Animación estimulativa para personas mayores. Narcea de Ediciones. Madrid. 2000

(3) GUTIERREZ, L. Métodos para la animación socio-cultural. Editorial CCS. Madrid. 1999

(4) JONES, D, SHEENA, E, HARTERY T, et al. Sociology and Occupational Therapy. Leisure. Chapter 14. Churchill Livingston. 1998

(5) MOSEY, A. Social Participation. 1996, p 340; In: YOUNGSTROM, Mary. The Occupational Therapy Practice Framework: The evolution of Our Professional Language. AJOT. November - December 2002; Volume 56. Number 6: 621

(6) YOUNGSTROM, Mary. The Occupational Therapy Practice Framework: The evolution of Our Professional Language. AJOT. November - December 2002; Volume 56. Number 6 : $607-629$ 